\title{
Commentary on article: Adrenal crisis in treated patients with Cushing's syndrome
}

\section{David J Torpy}

Endocrine and Metabolic Unit, Royal Adelaide Hospital, Adelaide, South Australia, Australia and Discipline of Medicine, University of Adelaide, Adelaide, South Australia, Australia
Correspondence should be addressed to D J Torpy

Email

david.torpy@sa.gov.au

\begin{abstract}
A study has examined the rates of adrenal crises in patients treated with pituitary or adrenal surgery. Rates were substantial (approximately 9 per 100 patient years), perhaps representing suppression of corticotrope ACTH secretion and deprivation of normal corticotrope number postoperatively. Hormone withdrawal syndrome may have contributed to the rates of apparent adrenal crises given the definition used. Higher rates were seen in patients given relatively high dose glucocorticoids postoperatively in one of the two centres where patients were treated perhaps some of the patients in the high dose centre had longer periods of corticotrope suppression from exogenous glucocorticoids, increasing the risk period for adrenal crises. The question of optimal glucocorticoid dose and weaning rate after cure of Cushing's syndrome remains a balance between weaning at a rate sufficiently rapid to allow resumption of normal corticotrope function thereby preventing adrenal crises and providing sufficient glucocorticoid support to avoid hormone withdrawal syndrome or even precipitating an adrenal crisis, in the vulnerable 4-6 month period after successful surgery. There is likely to be considerable inter-individual variability in optimum glucocorticoid dose and weaning rate so that close clinical and biochemical monitoring is currently a practical approach.
\end{abstract}

Broersen et al. have, in this issue of the journal, provided an interesting retrospective cohort analysis of adrenal crises in adult Cushing's syndrome patients ( $n=106$ over 15 years; 19 patients with 41 crises), after pituitary or adrenal surgery (74 vs $26 \%$ ) performed with at least initial cure of hypercortisolism (1). Adrenal crises (ACs) are a consequence of insufficient glucocorticoid tissue action. Glucocorticoid insufficiency may be absolute and follow from a lack of circulating cortisol or relative where low cortisol concentrations are below tissue requirements, particularly in times of inflammatory stress where the immunomodulatory effects of cortisol are critical for survival. ACs are all too frequent in patients with adrenal insufficiency at 6-8\% per annum, in patients with primary adrenal insufficiency and structural secondary insufficiency (2). Fortunately, given the high frequency of use of glucocorticoids for a myriad of disorders, ACs occur at much lower rates in patients who have glucocorticoidinduced adrenal insufficiency (3).

Adrenal crisis has no formal consensus definition, but, following the leadership of Prof. B Allolio, Broersen et al. defined ACs as episodes of acute health impairment requiring hospitalization and exhibiting prompt recovery after IV glucocorticoid, along with two of the diffuse features of acute adrenal insufficiency such as nausea, vomiting, severe fatigue, fever, somnolence, hyponatraemia or hyperkalaemia and hypotension $(1,4)$. Hypotension was not a mandatory inclusion criterion, although shock could be regarded as a relatively specific marker of an AC itself, if other causes of hypotension are excluded. Emergency room and self-treated episodes were excluded.

Patients successfully treated for pituitary or adrenal Cushing's with removal of an ACTH or cortisol secreting (c) 2019 European Society of Endocrinology Printed in Great Britain
Published by Bioscientifica Ltd. 
tumour, respectively, are clearly at risk of ACs. Most patients will have had prolonged hypercortisolism and have suppression of the hypothalamic-pituitary-adrenal axis, a phenomenon primarily involving suppression of corticotropin-releasing hormone (CRH) secretion from the hypothalamic paraventricular CRH neurons which regulate pituitary ACTH secretion after secretion into the hypothalamic-hypophyseal portal circulation. This assertion is based on the restoration of ACTH and cortisol secretion with exogenous CRH in hypocortisolaemic patients soon after pituitary adenomectomy for Cushing's disease (5).

Broersen et al. have described a risk of ACs that is comparable to patients with primary adrenal insufficiency and structural forms of secondary adrenal insufficiency, clearly higher than that in patients with HPA axis suppression for exogenous glucocorticoids, at approximately 9 per 100 patient years, with a somewhat higher risk in pituitary compared to adrenal surgery cases (9.6 vs 7.0 per 100 patient years) (1). AC risk was not evenly distributed among patients with one AC conferring an increased risk of a further episode, as for other forms of adrenal insufficiency $(1,2)$. AC risk was highest soon after surgery (median 1st crisis at 2 months, IQR 1-22 months) but episodes occurred after several years, suggesting corticotrope damage or prolonged exogenous suppression. AC risk factor analyses were limited by sample size but concomitant diabetes mellitus increased risk. Moreover, preoperative pituitary macroadenoma and postoperative multiple pituitary hormone deficits conferred higher AC risk, both factors that may indicate structural loss of corticotropes and consequent more complete cortisol deficiency.

An interesting observation involved the two centres studied - one in Leiden $(n=66)$ and the other in Berlin $(n=44)$. The risk of an AC was approximately double in Berlin (19 per 129 patient years) as compared to Leiden (22 per 328 patient years). The authors suggested that differences in patient education and general management may be relevant, although such differences could probably not be precisely defined. The risk factors for postCushing's ACs established in the study as a whole did not explain the AC rate difference - postoperative pituitary deficits, pituitary vs adrenal cases, diabetes mellitus frequency, were all similar. There was one difference Berlin patients were treated with approximately double the postoperative doses of hydrocortisone than the Leiden patients (median (IQR): Berlin-AC $50 \mathrm{mg}$ (30-65), Berlin-no AC $30 \mathrm{mg}$ (20-50); Leiden AC $20 \mathrm{mg}$ (0-35), Leiden no AC $20 \mathrm{mg}(20-40))$.
It may be that higher doses of hydrocortisone post cure of Cushing's above the physiological range (15$25 \mathrm{mg}$ daily) may suppress the HPA axis more completely and for longer than the usual recovery time postoperatively (4-12 months), rendering the patient more highly glucocorticoid dependent and hence more prone to severe glucocorticoid deficiency episodes. AC rates can be seen to mirror the severity of HPA axis defect in order from most severely to less severely deficient: primary adrenal insufficiency, secondary adrenal insufficiency and glucocorticoid-induced adrenal insufficiency. In accordance with this pattern, Cushing's patients who have had high-dose hydrocortisone post cure have both suppression from endogenous cortisol pre-op and suppression postoperatively. However, the rates of AC post Cushing's seem higher than might be expected part of this may be from structural loss of corticotropes from surgery as suggested by the association with macroadenomas/multiple pituitary hormone deficits.

Some diagnoses of ACs may represent, in part, episodes of hormone withdrawal syndrome, thought to be mediated by central opioid, dopamine mesocortico-limbic and noradrenergic pathways as well as inflammatory cytokine release leading to anorexia, nausea, emesis, weight loss, fatigue, myalgias, arthralgias, abdominal pain, lethargy and fever - all overlapping with the features of glucocorticoid deficiency but occurring with adequate glucocorticoid replacement (6). Hence, some ACs may have represented hormone withdrawal episodes due to the AC definition used and this could contribute to the high rates of apparent ACs, higher than that seen in patients taking/weaning exogenous glucocorticoids.

Finally, these data lead us to consider glucocorticoid administration after cure of Cushing's. There are no controlled data that point to an optimum regimen. Post-op physiological dosing with subsequent glucocorticoid complete wean over 4-12 months maximises rate of recovery from Cushing's. However, supraphysiological dosing minimises hormone withdrawal syndrome the cost may be slower recovery from Cushing's and perhaps even more ACs based on data from Broersen et al. Although still conjectural, one approach may be to treat with physiological doses of hydrocortisone post cure of Cushing's, but inform patients of the symptoms of hormone withdrawal as described earlier, and be ready to escalate glucocorticoid replacement doses in patients where the symptoms of withdrawal are intolerable. If symptoms are relatively mild they may be perceived as part of the healing process. On recovery of HPA axis function, typically in the 4- to 12-month postoperative interval, 
morning cortisol levels prior to AM hydrocortisone dosing may comprise a factor in dose reduction. A prospective study analysing the question of optimal postcure glucocorticoid management would need to be large as glucocorticoid withdrawal symptoms vary markedly in their type and severity between individuals. For the time being, close follow-up and individualized care are needed in the delicate postoperative period after surgical Cushing's cure.

\section{Declaration of interest}

The author declares that there is no conflict of interest that could be perceived as prejudicing the impartiality of this commentary.

\section{Funding}

This research did not receive any specific grant from any funding agency in the public, commercial or not-for-profit sector.

\section{References}

1 Broersen LHA, van Haalen FM, Kienitz T, Dekkers OM, Strasburger CJ, Periera AM \& Biermasz NR. Adrenal crisis in treated patients with Cushing's syndrome. European Journal of Endocrinology 2019181 201-210. (https://doi.org/10.1530/EJE-19-0202)

2 Rushworth RL, Torpy DJ \& Falhammar H. Adrenal crises: perspectives and research directions. Endocrine 201755 336-345. (https://doi. org/10.1007/s12020-016-1204-2)

3 Rushworth RL, Chrisp GL \& Torpy DJ. Glucocortioid-induced adrenal insufficiency: a study of the incidence in hospital patients and a review of post-operative management. Endocrine Practice 201824 437-445. (https://doi.org/10.4158/EP-2017-0117)

4 Allolio B. Extensive experience in endocrinology. Adrenal crisis. European Journal of Endocrinology 2015172 R115-R124.

5 Gomez MT, Magiakou MA, Mastorakos G \& Chrousos GP. The pituitary corticotroph is not the rate limiting step in the postoperative recovery of the hypothalamic-pituitary-adrenal axis in patients with Cushing's syndrome. Journal of Clinical Endocrinology and Metabolism 199377 173-177. (https://doi.org/10.1210/ jcem.77.1.8392083)

6 Hochberg Z, Pacak K \& Chrousos GP. Endocrine withdrawal syndromes. Endocrine Reviews 200324 523-538. (https://doi. org/10.1210/er.2001-0014)

Received 27 June 2019

Accepted 10 July 2019 